This item was submitted to Loughborough's Research Repository by the author.

Items in Figshare are protected by copyright, with all rights reserved, unless otherwise indicated.

\title{
How a process simulator and a rule-based system contribute to virtual reality applications for process safety
}

PLEASE CITE THE PUBLISHED VERSION

PUBLISHER

(C) Elsevier

VERSION

AM (Accepted Manuscript)

LICENCE

CC BY-NC-ND 4.0

\section{REPOSITORY RECORD}

Shang, Xiaolei, Paul Wai Hing Chung, Jeff Fry, Luca Vezzadini, and Mauro Colombo. 2019. "How a Process Simulator and a Rule-based System Contribute to Virtual Reality Applications for Process Safety". figshare. https://hdl.handle.net/2134/5731. 
This item was submitted to Loughborough's Institutional Repository (https://dspace.lboro.ac.uk/) by the author and is made available under the following Creative Commons Licence conditions.

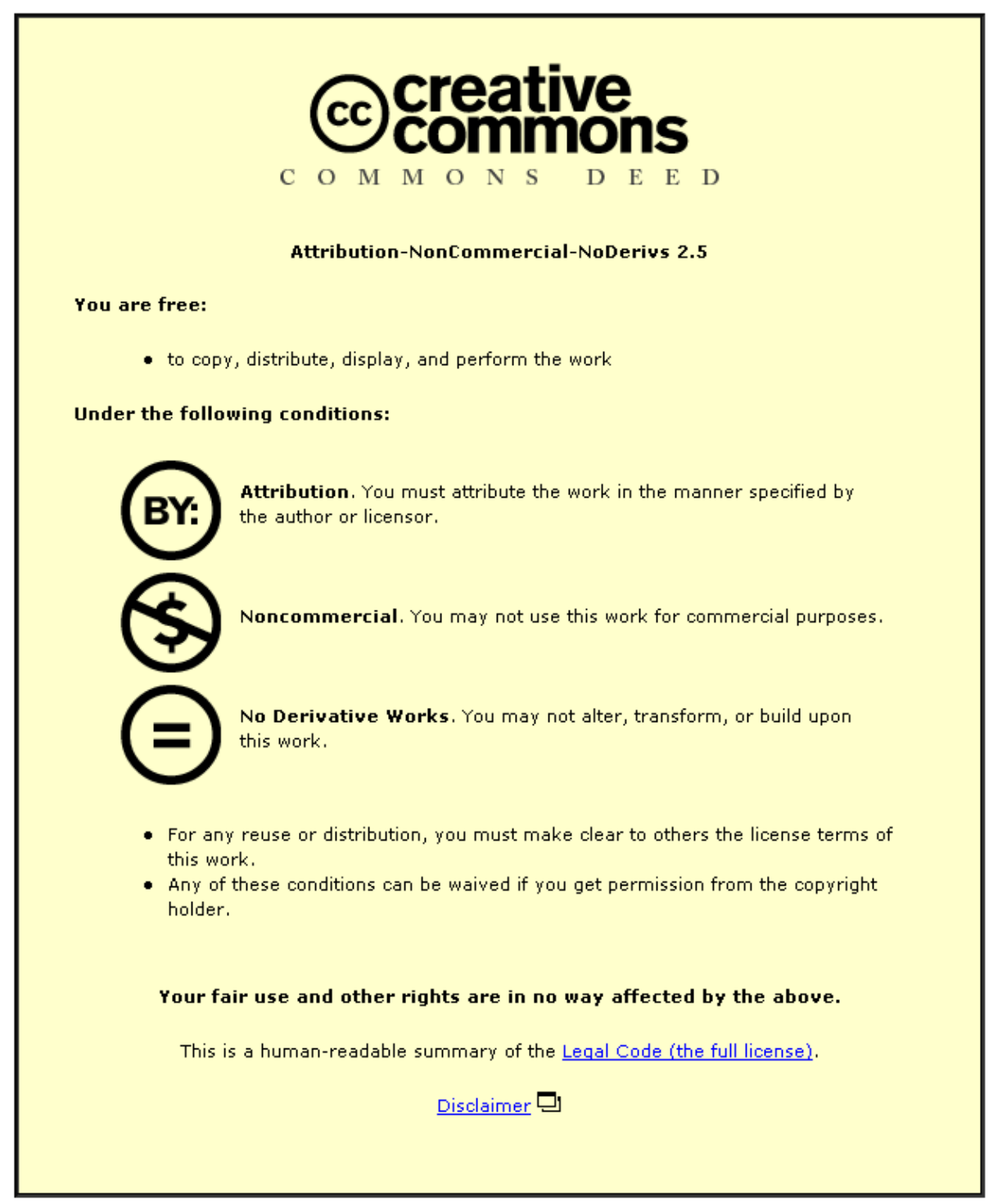

For the full text of this licence, please go to: http://creativecommons.org/licenses/by-nc-nd/2.5/ 


\title{
How a process simulator and a rule-based system contribute to virtual reality applications for process safety
}

\author{
Xiaolei Shang ${ }^{\mathrm{a}}$, Paul Chung ${ }^{\mathrm{a}}$, Jeff Fry ${ }^{\mathrm{a}}$ Luca Vezzadini $^{\mathrm{b}} \&$ Mauro Colombo $^{\mathrm{c}}$ \\ ${ }^{a}$ Department of Computer Science, Loughborough University, Loughborough LE11 3TU, United \\ Kingdom \\ ${ }^{b}$ Virtual Reality \& Multi Media Park, Corso Lombardia 190, Turin 10149 Italy \\ ${ }^{c}$ Politecnico di Milano, Dipartimento di Chimica, Materiali e Ingegneria Chimica "G. Natta", \\ Piazza Leonardo da Vinci 32, 20133 Milano Italy
}

\begin{abstract}
The VIRTHUALIS project aims to develop a number of virtual reality applications for improving safety in the process industries. The applications allow human factors experts to study how operators interact with plant, and provide a safe environment in which new safety actions can be tried and tested.

Safety applications are built on the SafeVR technology platform, a distributed client-server virtual reality system. This paper describes how two external modules - a process simulator and a rule-based system - are interfaced to the platform and the benefits they provide both separately and together. The two modules communicate with the platform's server by exchanging messages, conforming to a simple syntax.

pSimProxy provides a generic interface to an external process simulator, which in turn delivers the realistic plant behaviour. It handles bidirectional data exchange with and control of the external simulator. It can be configured at run time to use whichever available mechanisms are supported by the actual process simulator that models the plant being simulated.

ClipsClient is an expert or rule-based system, based on NASA's CLIPS expert system software that can make inferences about the information contained in the messages. It consists of a set of facts, a number of rules and an inference engine. It can be provided with a number of rules that monitor how operators are running the plant, and react in useful ways to these events.

The simulator notifies the server of changes in process parameters through a message. The values may be displayed, for example as gauge readings, in the virtual environment. As operators control the plant, their actions, say opening a valve, are also reported by messages via the server to the process simulation. Messages can also be read by the rule-based system, allowing it to maintain its own representation of the plant. This in turn permits automated expert reasoning on the state of the plant and the actions of its operators which can cause further message to be sent to the server. The rule-based system is therefore, a powerful mechanism for rapidly reconfiguring the application and general rules can be written that only require new facts at run-time to change the behaviour of the entire virtual environment.

The message syntaxes, the system architecture and the interfacing of the external modules are described along with examples showing their individual and joint benefits.
\end{abstract}

\section{Keywords: virtual reality, plant safety, process simulation, rule-based system}

\section{Introduction}

Safety is of enormous importance in many industries, especially those dealing with hazardous materials like the process industries. Virtual reality is an important visualisation technique being applied in plant safety [1]. The VIRTHUALIS project aims to develop a number of virtual reality applications for improving safety in the process industries. This will allow human factors experts to study how operators interact with plant, and provide a safe environment in which new safety actions can be tried and tested.

\subsection{The SafeVR platform}

Safety applications are built on the SafeVR technology platform (itself, originally based on Delta3D [2]), a distributed client-server system which manages a realistic, immersive, interactive, multi-user, 3D representation of a process plant, including:

- collision, gravity and motion models for realistic movement and navigation;

- environmental conditions, time of day, weather, etc.;

- 3D audio for environmental and spatially-located equipment sounds;

- controls to operate plant components (pumps, valves, PID controllers, etc.);

Paper published in the Proceedings of the 19th European Symposium on Computer Aided Process Engineering - ESCAPE 19 (Eds. Jezowski, J., \& Thullie, J.), European Symposium on Computer Aided Process Engineering - ESCAPE 19, Krakow, Poland, June 2009, pp.435-439, Elsevier, ISBN: 978-0-444-53433-0. 
- plant instrumentation (indicators and local panels etc.).

A typical deployment of the SafeVR platform elements, shown in Figure 1, consists of:-

- a master application (server) to co-ordinate and synchronise multiple user;

- one or more satellite applications (clients) that present individual, navigable views of the plant (or control room displays) to users;

- external applications (clients) that provide specific functionality;

- an efficient network to exchange messages between the applications.

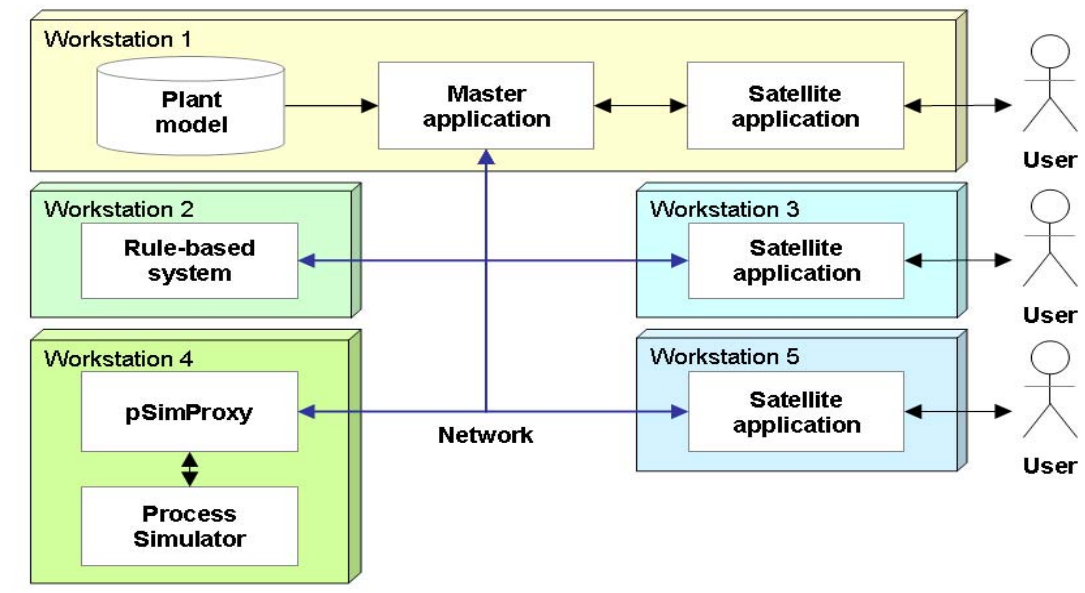

Figure 1 Architecture of the SafeVR platform

This paper describes two external applications and the messages they exchange with the server. pSimProxy is a generic interface to a process simulation, and ClipsClient is an interface to a rule-based system. Both use the Delta3D messaging mechanism and together they provide useful functionality.

\section{2. pSimProxy}

Although virtual reality provides an immersive 3D plant environment, an external process simulator provides the realistic chemical and physical behaviour of the plant. The simulation must be dynamic to respond to the operators in the virtual world. Unlike a_steady-state simulation, it responds to perturbations, and can model start-ups, shut-downs and exceptional conditions.

Plant owners usually have a suitable dynamic process simulation model: a survey of the industrial partners within VIRTHUALIS found several widely used simulators. To support a real-time virtual world, we identified those capable of fast, dynamic simulation, with refresh rates comparable to VR frame rates. From those, the following fairly generic set of features, were chosen for implementation:

- point-based representation of physical/chemical properties of a plant;

- getting, setting, polling and ramping point values;

- starting, stopping, pausing and resuming the simulation software;

- faster or slower than real-time simulation speeds,

- switching of simulation scenario, loading and saving simulation snapshots.

pSimProxy handles bidirectional data exchange with and control of the external simulator. It is configured at run time to use whichever APIs are supported by the actual process simulator being used. It read a list of points exposed by the process simulator, their data type and flow direction.

Data exchange and control of pSimProxy is provided by messages, as shown in Figure 2. The

messageProcessor parses incoming messages, extracting the message type, point(s) and action etc. It then calls pSimPoint and pSimControl methods which in turn invoke methods in the pSimAbstraction layer. This layer can also translate point names, mapping the SafeVR namespace to simulator point names and vice versa.

The pSimProxy provides the following functionality:

- setting process variables (pipe flows, tank levels etc.) in the simulator. As users operate the virtual plant's controls (open valves, start pumps etc.), changes are sent to the dynamic process simulator; 
- getting and polling of point values from the simulator, and tolerance. Point values are returned immediately or at a specified interval, if and only if the point value has changed by more than a tolerance since last reported;

- point ramping sets a target point value and slews to it over a specified time, simulating automated plant components and PID parameter controllers;

- timescale manipulation lets the user to "fast-forward" through periods of little interest or to examine other periods in "slow-motion".

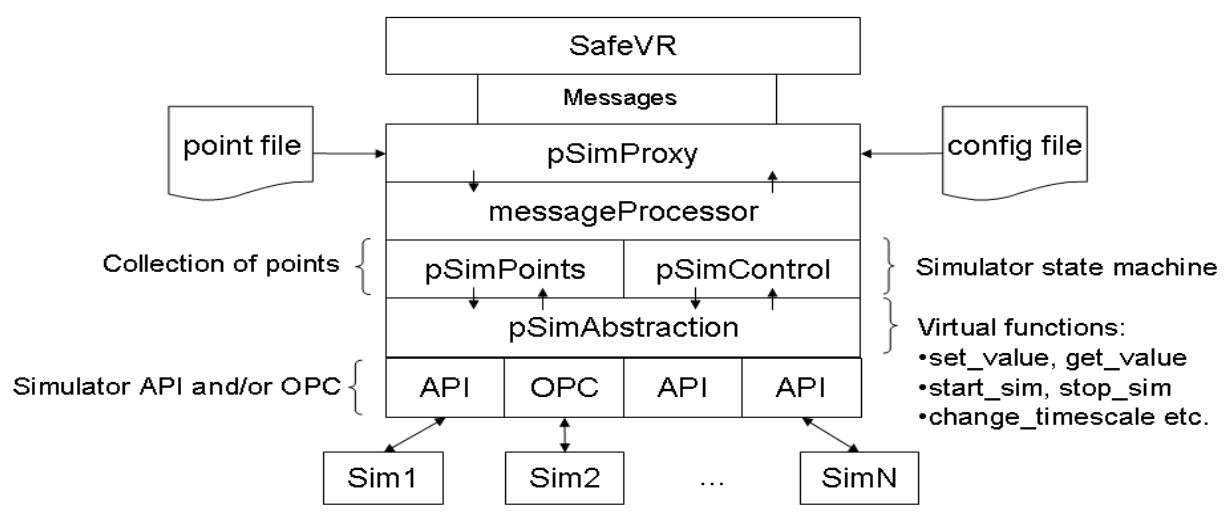

Figure 2 pSimProxy architecture

\section{ClipsClient}

An expert or rule-based system can monitor the information contained in the messages being exchanged by the process simulator and the server, to make inferences about the state of the plant, and how operators are controlling it.

ClipsClient is an interface to NASA's CLIPS expert system shell [3]. An expert system consists of a set of facts, a number of rules and an inference engine, allowing data and knowledge about a system to be represented separately. ClipsClient messageProcessor parses specific messages to extract updated point values, which it translates into a new CLIPS fact. The inference engine is then invoked to process the new fact using the current fact and rule set.

\section{Message types}

Communication, control and data flow between the master application and the external applications is implemented by six message types. Each message contains a string, composed of a number of token-value pairs as in Figure 3. This syntax is expressive, non-restrictive and easily extensible. The message types shown in figure 4 are:

- OPERATION_MESSAGE - sent by the master to all external applications whenever the user operates a plant component (e.g. opening a valve, switching off a pump etc.). The message specifies the component, a property and a new value;

- VALUE_UPDATE_MESSAGE - sent by pSimProxy to the master application to report a single updated point value;

- PSIM_POLL_MESSAGE - sent by the master to pSimProxy to set or change its polling behaviour (perpoint intervals or tolerances);

- PSIM_CONTROL_MESSAGE - sent by the master to pSimProxy to start or stop the process simulator, change the timescale, load or save point values;

- RULE_MESSAGE - sent by the rule-based system to the master application to return any results generated by its rules;

- VALUE_RELAY_MESSAGE - a VALUE_UPDATE_MESSAGE forwarded to ClipsClient, allowing it to respond.

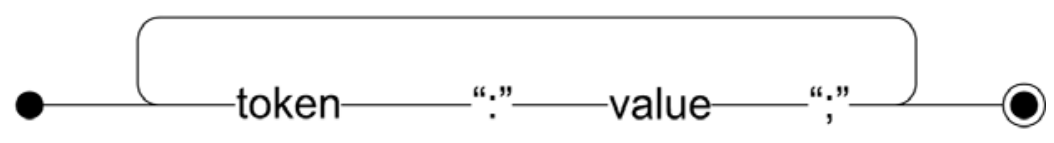

Figure 3 Syntax diagram of message content

Paper published in the Proceedings of the 19th European Symposium on Computer Aided Process Engineering - ESCAPE 19 (Eds. Jezowski, J., \& Thullie, J.), European Symposium on Computer Aided Process Engineering - ESCAPE 19, Krakow, Poland, June 2009, pp.435-439, Elsevier, ISBN: 978-0-444-53433-0. 


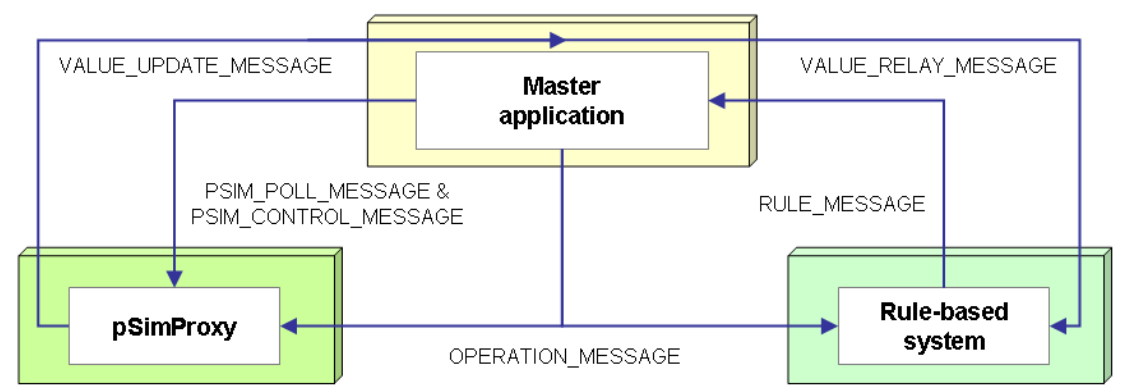

Figure 4 Messages exchanged by the Master application, Simulator and Rule-based Sysytem

\section{Examples of process simulator \& a rule-based system interaction}

Previous work [4] has described how CLIPS works with VIRTHUALIS applications. Several examples may help to demonstrate the contribution of the process simulator and rule-based system when used together.

Nominal sequences of actions (operations by users) can be modelled as rules, with actions in parallel, in any order, or with pre-requisite actions carried out beforehand. The sequence of operator actions can be monitored by the rule-based system, and a text-based tutor mode can give immediate operator feedback, for example as a training application.

Operating point values can be monitored by the rule-based system which can raise alarms, or trigger events such as gas leaks, fires or explosions. Faulty plant can be emulated: a stuck valve for example, can be created by a rule and fact. As the user tries to open the valve, the rule-based system intervenes to reset it to the 'sticky' maximum and the flow is realistically restricted.

\section{Conclusions and future work}

The combination of a process simulator and rule-based system provides dynamic, realistic plant behaviour, in response to operator actions, enriching safety applications and simplifing their construction. The VIRTHUALIS project continues to forsee their role in applications for design, training, risk assessment, accident investigation, operational safety management, even optimisation, during normal, unusual and exceptional operation.

\section{Acknowledgements}

VIRTHUALIS (www.virthualis.org) is supported financially by the European Commission. The authors are very grateful for the technical support of Invensys.

\section{References}

[1] P. Cozens, J. Waters and R. Neale, A virtual reality approach to personal safety and the design of built environment facilities. The conference proceedings of the $18^{\text {th }}$ ARCOM Annual Conference, Volume 2 (2003) pp 461-473.

[2] www.delta3d.org

[3] J. C. Giarratano \& G. Riley, Expert Systems: Principals and Programming. Third Edition. PWS Publishing Company, Boston MA. USA. (1998) ISBN: 0-534-95053-1.

[4] P. W. H. Chung, X. Shang, Z. Nivolianitou and J. D. Fry, Improving Process Safety Training Through the Use of VR and Knowledge-based Technologies, Proceedings of the 17th ARRTS - Advances in Risk and Reliability Technology Symposium, L Bartlett (ed), Loughborough University (April 2007) pp 394-400 ISBN 0904947629. 